\title{
Oscillatory Behaviour of the Nonlinear Damped Fractional Partial Dynamic Equation
}

\author{
R. Ramesh, ${ }^{1}$ S. Harikrishnan, ${ }^{2}$ P. Prakash, ${ }^{3}$ and Yong-Ki Ma ${ }^{4}{ }^{4}$ \\ ${ }^{1}$ Department of Mathematics, Muthayammal College of Engineering, Rasipuram 637408, India \\ ${ }^{2}$ Department of Mathematics, Sona College of Technology, Salem 636005, India \\ ${ }^{3}$ Department of Mathematics, Periyar University, Salem 636011, India \\ ${ }^{4}$ Department of Applied Mathematics, Kongju National University, Chungcheongnam-do 32588, Republic of Korea
}

Correspondence should be addressed to Yong-Ki Ma; ykma@kongju.ac.kr

Received 2 September 2021; Revised 20 December 2021; Accepted 27 January 2022; Published 23 February 2022

Academic Editor: Maria L. Gandarias

Copyright $\odot 2022$ R. Ramesh et al. This is an open access article distributed under the Creative Commons Attribution License, which permits unrestricted use, distribution, and reproduction in any medium, provided the original work is properly cited.

In this paper, a partial dynamic equation of fractional order is considered with Neumann and Dirichlet boundary conditions, and we studied the oscillation properties of the fractional partial dynamic equation on time scales. Riccati transformation technique is used to establish oscillation criteria for the fractional partial dynamic equation. The obtained results are verified with examples.

\section{Introduction}

In recent years, the importance of fractional-order calculus has been much motivated by researchers rather than the integer order because of increasing applications in signal processing, neural networks, and electrical and mechanical engineering. Definitions for fractional derivatives in continuous and discrete cases using various operators and functions have been given by many such as Hadamard, Euler, Riemann, and Grunwald, but fundamental properties of derivatives were not satisfied by these derivatives, and with these derivatives, solving the differential equations of fractional order is not easy. In [1], the definition of the conformable fractional derivative has been given, and it has satisfied some fundamental properties of derivatives. The properties and application of the conformable derivative have been presented in $[2,3]$ and the references cited therein. Meanwhile, the oscillation properties of solutions are an important qualitative tool to study the solutions of dynamical systems. The oscillation of different types of both integer-order and fractional-order differential, difference, and dynamic equations has been investigated by many authors [4-20]. The time-scale calculus was introduced by Stefan Hilger to unify the theory of continuous and discrete cases. The time-scale analysis for multivariable cases is discussed in [21-23], and the fractional time-scale calculus is studied in [24]. To the best of our knowledge, we observe that the oscillation of solutions of the fractional partial dynamic equation on the time scale was not considered so far.

This motivates the authors to establish oscillation results of the following fractional partial dynamic equation with the damped term:

$$
\left(\ell(\eta) \chi^{\left(\Delta^{\alpha}\right)_{\eta}}(\xi, \eta)\right)^{\left(\Delta^{\alpha}\right)_{\eta}}+\hbar(\eta) \chi^{\left(\Delta^{\alpha}\right)_{\eta}}(\xi, \eta)+v(\xi, \eta) f(\chi(\xi, \eta))=\tau(\eta) \nabla_{\xi}^{2} \chi(\xi, \eta),
$$

where $(\xi, \eta) \in \Omega \times\left[\eta_{0}, \infty\right)_{\Theta}$ with the Neumann boundary condition

$$
\frac{\partial \chi(\xi, \eta)}{\partial \nu}=0, \quad(\xi, \eta) \in \partial \Omega \times\left[\eta_{0}, \infty\right)_{\Theta}
$$


and the Dirichlet boundary condition

$$
\chi(\xi, \eta)=0, \quad(\xi, \eta) \in \partial \Omega \times\left[\eta_{0}, \infty\right)_{\Theta},
$$

where $\left(\Delta^{\alpha}\right)_{\eta}$ is the conformable fractional partial dynamic operator with respect to the time variable $\eta, \Omega$ is a bounded domain in $\mathbb{R}^{n}$ with piecewise smooth boundary $\partial \Omega, v$ is the unit exterior normal vector to $\partial \Omega$ and $\alpha \in(0,1]$, and $\nabla_{\xi}^{2}=$ $\sum_{i=1}^{n} \partial_{\xi_{\xi} \xi_{i}}^{2}$ is the Laplacian operator. $\ell(\eta), \hbar(\eta)$, and $\tau(\eta)$ are real-valued and rd-continuous functions on $[0, \infty)_{\Theta}, \ell(\eta)>0$ is conformable fractional differentiable with $\ell(\eta)^{\left(\Delta^{\alpha}\right)_{\eta}}$ continuous, and $v(\xi, \eta) \in C\left(\bar{\Omega} \times\left[\eta_{0}, \infty\right)_{\Theta}, \mathbb{R}\right)$ with $v(\eta)=\min _{\xi \in \Omega}$ $v(\xi, \eta)$. The continuous function $f$ satisfies $\chi f(\chi)>0$ for $\chi \neq 0$.

A time scale $\Theta$ is a nonempty closed subset of the real numbers $\mathbb{R}$ which is unbounded above. The time-scale interval of the form $\left[\eta_{0}, \infty\right)_{\Theta}$ is denoted by $\left[\eta_{0}, \infty\right)_{\Theta}=\left[\eta_{0}\right.$, $\infty) \cap \Theta$ for $\eta_{0} \in \Theta, \eta_{0}>0$.

A nontrivial solution $\chi(\xi, \eta)$ which satisfies (1) on $\Omega \times$ $\left[\eta_{0}, \infty\right)_{\Theta}$ along with boundary condition (2) (or (3)) is called oscillatory if it has neither eventually positive solution (EPS) nor eventually negative solution (ENS). Equation (1) is oscillatory if all the solutions of it are oscillatory.

\section{Preliminaries}

The following definitions can be found in [25], where there is a detailed introduction to time-scale calculus. A time scale $\Theta$ is a nonempty closed subset of the real numbers $\mathbb{R}$. We will use intervals of the form $\left[t_{0}, \infty\right)_{\Theta}=\left[t_{0}, \infty\right) \cap \Theta$ for $t_{0} \in \Theta$. For a point $t \in \Theta$, we have the following definitions: the forward jump operator is defined as $\sigma(t)=\inf \{s \in \Theta, s>t\}$. The backward jump operator is defined as $\rho(t)=\sup \{s \in \Theta, s<t\}$. The graininess is defined as $\mu(t)=\sigma(t)-t$. A point $t \in \Theta$ is said to be right dense if $\sigma(t)=t$. A function $g: \Theta \longrightarrow \mathbb{R}$ is said to be rd-continuous if it is continuous at each right-dense point and there exists a finite left limit of $g$ at all left-dense points. The set of rd-continuous functions is denoted by $C_{\mathrm{rd}}(\Theta, \mathbb{R})$.

To define derivatives, we introduce

$$
\Theta^{k}= \begin{cases}\Theta \backslash(\rho(\sup \Theta), \sup \Theta) & \text { if } \sup \Theta<\infty \\ \Theta & \text { if } \sup \Theta<\infty\end{cases}
$$

Definition 1 (see [24]). Let $f: \Theta \longrightarrow \mathbb{R} ; \eta \in \Theta^{k}$, and $\alpha \in$ $(0,1]$. For $\eta>0$, we define $f^{\left(\Delta^{\alpha}\right)}(\eta)$ to be the number (provided it exists) with the property that, given any $\epsilon>0$, there is a $\delta$-neighborhood $\mathcal{N}_{\eta} \in \Theta$ of $\eta, \delta>0$ such that

$$
\left|\left[f^{\sigma}(\eta)-f(\kappa)\right] \eta^{1-\alpha}-f^{\left(\Delta^{\alpha}\right)}(\eta)[\sigma(\eta)-\kappa]\right| \leq \varepsilon|\sigma(\eta)-\kappa| \text { for all } \kappa \in \mathcal{N}_{\eta}
$$

$f^{\left(\Delta^{\alpha}\right)}(\eta)$ is called the conformable fractional derivative of $f$ of order $\alpha$ at $\eta$, and the conformable fractional derivative at 0 is $f^{\left(\Delta^{\alpha}\right)}(0)=\lim _{\eta \longrightarrow 0^{+}} f^{\left(\Delta^{\alpha}\right)}(\eta)$.
Lemma 1. Let $a \in \Theta^{k}$ and $b \in \Theta$, and assume that $f: \Theta \times$ $\Theta^{k} \longrightarrow \mathbb{R}$ is continuous at $(\eta, \eta)$ where $\eta \in \Theta^{k}$ with $a<\eta$. Suppose that, for all $\epsilon>0$, there is a $\delta$-neighborhood $U$ of $\eta_{i}$ such that

$$
\left|\left[f^{\sigma_{i}}(\eta)-f_{i}^{\kappa}(\eta)\right] \eta_{i}^{1-\alpha}-f^{\left(\Delta^{\alpha}\right)_{\eta_{i}}}(\eta)\left[\sigma_{i}(\eta)-\kappa\right]\right| \leq \varepsilon\left|\sigma_{i}(\eta)-\kappa\right| \text { for all } \kappa \in U
$$

where $f^{\left(\Delta^{\alpha}\right) \eta_{i}}$ denotes the partial derivative of $f$ with respect to $\eta_{i}$. Then,

$$
\begin{aligned}
& g(\eta)=\int_{a}^{\eta} f(\eta, \kappa) \Delta \kappa \Rightarrow g^{\left(\Delta^{\alpha}\right)_{\eta_{i}}}(\eta)=\int_{a}^{\eta} f^{\left(\Delta^{\alpha}\right)_{\eta_{i}}}(\eta, \kappa) \Delta \kappa+f\left(\sigma_{i}(\eta), \eta\right), \\
& h(\eta)=\int_{\eta}^{b} f(\eta, \kappa) \Delta \kappa \Rightarrow h^{\left(\Delta^{\alpha}\right) \eta_{i}}(\eta)=\int_{\eta}^{b} f^{\left(\Delta^{\alpha}\right) \eta_{i}}(\eta, \kappa) \Delta \kappa-f\left(\sigma_{i}(\eta), \eta\right) .
\end{aligned}
$$

Proof. The proof is analogous to the proof of Theorem 1.117 in [25].

Definition 2. Let $\mathbb{B}=\left\{(\eta, \kappa) \mid \eta \geq \kappa \geq \eta_{0}\right\}$. Then, the class $\Lambda$ is a collection of functions $\Lambda \in C_{\mathrm{rd}}(\mathbb{B}, \mathbb{R})$ such that $\Lambda(\eta, \eta)=0$ for $\eta \geq \eta_{0}, \Lambda(\eta, \kappa)>0$ for $\eta>\kappa \geq \eta_{0}$, and $\Lambda$ has a nonpositive continuous $\alpha$-partial fractional derivative $\Lambda^{\left(\Delta^{\alpha}\right)_{\kappa}}(\eta, \kappa)$.

A function $g: \Theta \longrightarrow \mathbb{R}$ is said to be regressive provided $1+\mu(t) g(t) \neq 0$ for each $t \in \Theta^{k}$. Let $\mathscr{R}$ be the set of functions that are rd-continuous and regressive. Also, we define 
$\mathscr{R}^{+}=\{g \in \mathscr{R}: 1+\mu(t) g(t)>0, t \in \Theta\}$. For $\gamma \in \mathscr{R}$ and $s, t \in \Theta$, the generalized exponential function is defined by

$$
e_{\gamma}(t, s)=\exp \left(\int_{s}^{t} \zeta_{\mu(\tau)}(\gamma(\tau)) \Delta \tau\right), \quad \zeta_{\mu}(z)= \begin{cases}\frac{1}{\mu} \log (1+\mu z), & \mu \neq 0 \\ z, & \mu=0\end{cases}
$$

For convenience, we use the following:

$$
\begin{aligned}
& \Upsilon(\eta)=\int_{\Omega} \chi(\xi, \eta) \mathrm{d} \xi \\
& R(\eta)=-\frac{\eta^{\alpha-1} \hbar(\eta)}{\ell(\eta)}
\end{aligned}
$$

\section{Oscillation of (1) with Boundary Condition (2)}

Lemma 2. If the fractional dynamic inequalities

$$
\begin{aligned}
& {\left[\ell(\eta) \Upsilon^{\left(\Delta^{\alpha}\right)}(\eta)\right]^{\left(\Delta^{\alpha}\right)}+\hbar(\eta) \Upsilon^{\left(\Delta^{\alpha}\right)}(\eta)+v(\eta) f(\Upsilon(\eta)) \leq 0, \eta \geq \eta_{1}} \\
& {\left[\ell(\eta) \Upsilon^{\left(\Delta^{\alpha}\right)}(\eta)\right]^{\left(\Delta^{\alpha}\right)}+\hbar(\eta) \Upsilon^{\left(\Delta^{\alpha}\right)}(\eta)+v(\eta) f(\Upsilon(\eta)) \geq 0, \eta \geq \eta_{1}}
\end{aligned}
$$

have no EPS and ENS, respectively, then every solution $\chi(\xi, \eta)$ of (1) and (2) is oscillatory.
Proof. Suppose that $\chi(\xi, \eta)$ is an EPS of (1) and (2). Then, by the definition of EPS, there exists $\eta_{1} \geq \eta_{0}$ such that $\chi(\xi, \eta)>0$ for $\eta \geq \eta_{1}$, which gives $\Upsilon(\eta)>0$ for $\eta \geq \eta_{1}$. Now, taking integration to (1) in connection with $\xi$ over $\Omega$, we get

$$
\begin{aligned}
\int_{\Omega} & {\left[\ell(\eta) \chi^{\left(\Delta^{\alpha}\right)_{\eta}}(\xi, \eta)\right]^{\left(\Delta^{\alpha}\right)_{\eta}} \mathrm{d} \xi+\int_{\Omega} \hbar(\eta) \chi^{\left(\Delta^{\alpha}\right)_{\eta}}(\xi, \eta) \mathrm{d} \xi+\int_{\Omega} v(\xi, \eta) f(\chi(\xi, \eta)) \mathrm{d} \xi } \\
= & \int_{\Omega} \tau(\eta) \nabla_{\xi}^{2} \chi(\xi, \eta) \mathrm{d} \xi .
\end{aligned}
$$

Applying Green's formula and (2), we get

$$
\int_{\Omega} \nabla_{\xi}^{2} \chi(\xi, \eta) \mathrm{d} \xi=\int_{\partial \Omega} \frac{\partial \chi(\xi, \eta)}{\partial \nu} \mathrm{d} S=0
$$

By using the condition on $v$ and applying Jensen's inequality, we obtain

$$
\int_{\Omega} v(\xi, \eta) f(\chi(\xi, \eta)) \mathrm{d} \xi \geq v(\eta) \int_{\Omega} f(\chi(\xi, \eta)) \mathrm{d} \xi \geq v(\eta) f(\Upsilon(\eta)) .
$$

From (12)-(14), we have

$$
\begin{aligned}
& \int_{\Omega}\left[\ell(\eta) \chi^{\left(\Delta^{\alpha}\right)_{\eta}}(\xi, \eta)\right]^{\left(\Delta^{\alpha}\right)_{\eta}} \mathrm{d} \xi \\
& \quad+\hbar(\eta) \int_{\Omega} \chi^{\left(\Delta^{\alpha}\right)_{\eta}}(\xi, \eta) \mathrm{d} \xi+v(\eta) f(\Upsilon(\eta)) \leq 0 .
\end{aligned}
$$

Using that $\left[\ell(\eta) \chi^{\left(\Delta^{\alpha}\right)_{\eta}}(\xi, \eta)\right]^{\left(\Delta^{\alpha}\right)_{\eta}}$ is continuous in connection with $\xi$ on the closed and bounded set $\bar{G} \subseteq \mathscr{R}^{n}$, we have $\delta$ independent of $\eta$ in Lemma 1 . Therefore, the conditions of Lemma 1 are satisfied. Thus, $\int_{G}[\chi(\xi$, $\eta) \mathrm{d} \xi]^{\left(\Delta^{\alpha}\right)_{\eta}}=\int_{G} \chi^{\left(\Delta^{\alpha}\right)_{\eta}}(\xi, \eta) \mathrm{d} \xi$. Hence, from (15), we obtain (10). A similar argument is used for the $\operatorname{ENS} \chi(\xi, \eta)$ of (1)and (2) to obtain (11).

Lemma 3. Assume that $\int_{\eta}^{\infty} \Delta^{\alpha} \kappa / \ell(\kappa)=\infty$ and $R(\eta) \in \mathscr{R}_{+}$. If (10) has an $\operatorname{EPS} \Upsilon(\eta)$, then there exists sufficiently large $\eta_{1} \in\left[\eta_{0}, \infty\right)_{\Theta}$ such that $\left[\ell(\eta) \Upsilon^{\left(\Delta^{\alpha}\right)}(\eta) / e_{R(\eta)}\left(\eta, \eta_{0}\right)\right]^{\left(\Delta^{\alpha}\right)}<0$ and $Y^{\left(\Delta^{\alpha}\right)}(\eta)>0$ on $[\eta, \infty)_{\Theta}$.

Proof. Given $\Upsilon(\eta)$ is an EPS of (10), there is sufficiently large $\eta_{2}$ so that $\Upsilon(\eta)>0$ on $[\eta, \infty)_{\Theta}$. Now, we have 


$$
\begin{aligned}
{\left[\frac{\ell(\eta) \Upsilon^{\left(\Delta^{\alpha}\right)}(\eta)}{e_{R(\eta)}\left(\eta, \eta_{0}\right)}\right]^{\left(\Delta^{\alpha}\right)} } & =\frac{e_{R(\eta)}\left(\eta, \eta_{0}\right)\left[\ell(\eta) \Upsilon^{\left(\Delta^{\alpha}\right)}(\eta)\right]^{\left(\Delta^{\alpha}\right)}-\ell(\eta) \Upsilon^{\left(\Delta^{\alpha}\right)}(\eta)\left[e_{R(\eta)}\left(\eta, \eta_{0}\right)\right]^{\left(\Delta^{\alpha}\right)}}{e_{R(\eta)}\left(\eta, \eta_{0}\right) e_{R(\eta)}\left(\sigma(\eta), \eta_{0}\right)} \\
& =\frac{e_{R(\eta)}\left(\eta, \eta_{0}\right)\left[\ell(\eta) \Upsilon^{\left(\Delta^{\alpha}\right)}(\eta)\right]^{\left(\Delta^{\alpha}\right)}+\hbar(\eta) \Upsilon^{\left(\Delta^{\alpha}\right)}(\eta) e_{R(\eta)}\left(\eta, \eta_{0}\right)}{e_{R(\eta)}\left(\eta, \eta_{0}\right) e_{R(\eta)}\left(\sigma(\eta), \eta_{0}\right)} \\
& =\frac{-v(\eta) f(\Upsilon(\eta))}{e_{R(\eta)}\left(\sigma(\eta), \eta_{0}\right)}<0 .
\end{aligned}
$$

Since $R(\eta) \in \mathbb{R}_{+}$, we get $e_{R(\eta)}\left(\sigma(\eta), \eta_{0}\right)>0$, and $\ell(\eta)$ $\Upsilon^{\left(\Delta^{\alpha}\right)}(\eta) / e_{R(\eta)}\left(\eta, \eta_{0}\right)$ is decreasing on $\left[\eta_{2}, \infty\right)_{\Theta}$. Therefore, we get that $\Upsilon^{\left(\Delta^{\alpha}\right)}(\eta)$ is the EPS or ENS. Suppose that $\Upsilon^{\left(\Delta^{\alpha}\right)}(\eta)<0$ on $\left[\eta_{2}, \infty\right)_{\Theta}$ for sufficiently large $\eta_{2}>\eta_{1}$. Then,

$\Upsilon(\eta)-\Upsilon\left(\eta_{3}\right)=\int_{\eta_{2}}^{\eta} \frac{\ell(\kappa) \Upsilon^{\left(\Delta^{\alpha}\right)}(\kappa)}{\ell(\kappa)} \Delta^{\alpha} \kappa \leq \ell\left(\eta_{2}\right) \Upsilon^{\left(\Delta^{\alpha}\right)}\left(\eta_{2}\right) \int_{\eta_{2}}^{\eta} \frac{\Delta^{\alpha} \kappa}{\ell(\kappa)}$.

Letting $\eta \longrightarrow \infty$ and using the condition that $\int_{\eta}^{\infty} \Delta^{\alpha} \kappa / \ell(\kappa)=\infty$, we get $\lim _{\eta \rightarrow \infty} \Upsilon(\eta)=-\infty$ which is a contradiction to $\Upsilon(\eta)>0$, and hence, $\Upsilon^{\left(\Delta^{\alpha}\right)}(\eta)>0$.

The following lemma holds if we proceed as in the above lemma with an $\operatorname{ENS} \Upsilon(\eta)$.

Lemma 4. Assume that $\int_{\eta}^{\infty} \Delta^{\alpha} \kappa / \ell(\kappa)=\infty$ and $R(\eta) \in \mathscr{R}_{+}$. If (11) has an ENS $\Upsilon(\eta)$, then there exists sufficiently large $\eta_{1} \in\left[\eta_{0}, \infty\right)_{\Theta}$ such that $\left[\ell(\eta) \Upsilon^{\left(\Delta^{\alpha}\right)}(\eta) / e_{R(\eta)}\left(\eta, \eta_{0}\right)\right]^{\left(\Delta^{\alpha}\right)}>0$ and $Y^{\left(\Delta^{\alpha}\right)}(\eta)<0$ on $[\eta, \infty)_{\Theta}$.
Theorem 1. Assume that $\int_{\eta_{0}}^{\infty} \Delta^{\alpha} \kappa / \ell(\kappa)=\infty, R(\eta) \in \mathscr{R}_{+}$, and $f^{\prime}$ exists with $f^{\prime}(\xi) \geq N$ for some $N>0, \forall \xi \neq 0$. If there exists $z(\eta)>0$ on $[H, \infty)_{\Theta}$ such that

$$
\limsup _{\eta \rightarrow \infty} \int_{H}^{\eta}\left[\frac{v(\kappa) z(\kappa)}{e_{R(\kappa)}\left(\sigma(\kappa), \eta_{0}\right)}-\frac{\ell(\kappa)\left(z^{\left(\Delta^{\alpha}\right)}(\kappa)\right)^{2}}{4 N z(\kappa) e_{R(\eta)}\left(\kappa, \eta_{0}\right)}\right] \Delta^{\alpha} \kappa=\infty,
$$

then (1) and (2) are oscillatory.

Proof. Suppose that the solution $\chi(\xi, \eta)$ of (1) and (2) is the EPS. Define a generalized Riccati function as

$$
w(\eta)=\left[\frac{z(\eta) \ell(\eta) \Upsilon^{\left(\Delta^{\alpha}\right)}(\eta)}{f[\Upsilon(\eta)] e_{R(\eta)}\left(\eta, \eta_{0}\right)}\right] .
$$

By Lemma 3, it is lucid that $\Upsilon>0$ and $\Upsilon^{\left(\Delta^{\alpha}\right)} \geq 0$, respectively. Hence, $w(\eta) \geq 0$ for $\eta \geq \eta_{1}$. Then,

$$
\begin{aligned}
w^{\left(\Delta^{\alpha}\right)}(\eta)= & \frac{z(\eta)}{f[\Upsilon(\eta)]}\left[\frac{e_{R(\eta)}\left(\eta, \eta_{0}\right)\left[\ell(\eta) \Upsilon^{\left(\Delta^{\alpha}\right)}(\eta)\right]^{\left(\Delta^{\alpha}\right)}-\left(e_{R(\eta)}\left(\eta, \eta_{0}\right)\right)^{\left(\Delta^{\alpha}\right)} \ell(\eta) \Upsilon^{\left(\Delta^{\alpha}\right)}(\eta)}{e_{R(\eta)}\left(\eta, \eta_{0}\right) e_{R(\eta)}\left(\sigma(\eta), \eta_{0}\right)}\right] \\
& +\frac{\ell(\sigma(\eta)) \Upsilon^{\left(\Delta^{\alpha}\right)}(\sigma(\eta))}{e_{R(\eta)}\left(\sigma(\eta), \eta_{0}\right)}\left[\frac{f\left(\Upsilon(\eta) z^{\left(\Delta^{\alpha}\right)}(\eta)-z(\eta)[f(\Upsilon(\eta))]^{\left(\Delta^{\alpha}\right)}\right.}{f[\Upsilon(\eta)] f[\Upsilon(\sigma(\eta)]}\right] \\
\leq & \frac{-v(\eta) z(\eta)}{e_{R(\eta)}\left(\sigma(\eta), \eta_{0}\right)}+\frac{z^{\left(\Delta^{\alpha}\right)}(\eta) w(\sigma(\eta))}{z(\sigma(\eta))}-\frac{N z(\eta) e_{R(\eta)}\left(\eta, \eta_{0}\right)}{\ell(\eta)}\left[\frac{w(\sigma(\eta))}{z(\sigma(\eta))}\right]^{2} \\
\leq & \frac{-v(\eta) z(\eta)}{e_{R(\eta)}\left(\sigma(\eta), \eta_{0}\right)}+\frac{\ell(\eta)\left(z^{\left(\Delta^{\alpha}\right)}(\eta)\right)^{2}}{4 N z(\eta) e_{R(\eta)}\left(\eta, \eta_{0}\right)}
\end{aligned}
$$

By taking $\alpha$-integration from $\eta_{1}$ to $\eta$, we obtain

$$
\begin{aligned}
& \limsup _{\eta \rightarrow \infty} \int_{\eta_{1}}^{\eta}\left[\frac{v(\kappa) z(\kappa)}{e_{R(\kappa)}\left(\sigma(\kappa), \eta_{0}\right)}-\frac{\ell(\kappa)\left(z^{\left(\Delta^{\alpha}\right)}(\kappa)\right)^{2}}{4 N z(\kappa) e_{R(\eta)}\left(\kappa, \eta_{0}\right)}\right] \Delta^{\alpha} \kappa \\
& \quad \leq w\left(\eta_{1}\right)-w(\eta) \leq w\left(\eta_{1}\right) \leq \infty,
\end{aligned}
$$

which is a contradiction to (18). Similarly, we get a contradiction while assuming that $\chi(\xi, \eta)$ is the ENS of (1) and (2).

Theorem 2. Assume that $\int_{\eta}^{\infty} \Delta^{\alpha} \kappa / \ell(\kappa)=\infty, R(\eta) \in \mathscr{R}_{+}$, and $f(\xi) \geq E x$ for some $E>0, \forall \xi \neq 0$. If there exists $z(\eta)>0$ on $[H, \infty)_{\Theta}$ such that 
$\limsup _{\eta \rightarrow \infty} \int_{H}^{\eta}\left[\frac{E v(\kappa) z(\kappa)}{e_{R(\kappa)}\left(\sigma(\kappa), \eta_{0}\right)}-\frac{\ell(\kappa)\left(z^{\left(\Delta^{\alpha}\right)}(\kappa)\right)^{2}}{4 z(\kappa) e_{R(\eta)}\left(\kappa, \eta_{0}\right)}\right] \Delta^{\alpha} \kappa=\infty$,

then (1) and (2) are oscillatory.
Proof. Suppose that the solution $\chi(\xi, \eta)$ of (1) and (2) is the EPS. Define a generalized Riccati function as

$$
w(\eta)=\left[\frac{z(\eta) \ell(\eta) \Upsilon^{\left(\Delta^{\alpha}\right)}(\eta)}{\Upsilon(\eta) e_{R(\eta)}\left(\eta, \eta_{0}\right)}\right] .
$$

By Lemma 3, it is lucid that $\Upsilon>0$ and $\Upsilon^{\left(\Delta^{\alpha}\right)} \geq 0$, respectively. Hence, $w(\eta) \geq 0$ for $\eta \geq \eta_{1}$. Then,

$$
\begin{aligned}
w^{\left(\Delta^{\alpha}\right)}(\eta)= & \frac{z(\eta)}{\Upsilon(\eta)}\left[\frac{e_{R(\eta)}\left(\eta, \eta_{0}\right)\left[\ell(\eta) \Upsilon^{\left(\Delta^{\alpha}\right)}(\eta)\right]^{\left(\Delta^{\alpha}\right)}-\left(e_{R(\eta)}\left(\eta, \eta_{0}\right)\right)^{\left(\Delta^{\alpha}\right)} \ell(\eta) \Upsilon^{\left(\Delta^{\alpha}\right)}(\eta)}{e_{R(\eta)}\left(\eta, \eta_{0}\right) e_{R(\eta)}\left(\sigma(\eta), \eta_{0}\right)}\right] \\
& +\frac{\ell(\sigma(\eta)) \Upsilon^{\left(\Delta^{\alpha}\right)}(\sigma(\eta))}{e_{R(\eta)}\left(\sigma(\eta), \eta_{0}\right)}\left[\frac{\Upsilon(\eta) z^{\left(\Delta^{\alpha}\right)}(\eta)-z(\eta)[\Upsilon(\eta)]^{\left(\Delta^{\alpha}\right)}}{\Upsilon(\eta) \Upsilon(\sigma(\eta)}\right] \\
\leq & \frac{-E v(\eta) z(\eta)}{e_{R(\eta)}\left(\sigma(\eta), \eta_{0}\right)}+\frac{z^{\left(\Delta^{\alpha}\right)}(\eta) w(\sigma(\eta))}{z(\sigma(\eta))}-\frac{z(\eta) e_{R(\eta)}\left(\eta, \eta_{0}\right)}{\ell(\eta)}\left[\frac{w(\sigma(\eta))}{z(\sigma(\eta))}\right]^{2} \\
\leq & \frac{-E v(\eta) z(\eta)}{e_{R(\eta)}\left(\sigma(\eta), \eta_{0}\right)}+\frac{\ell(\eta)\left(z^{\left(\Delta^{\alpha}\right)}(\eta)\right)^{2}}{4 z(\eta) e_{R(\eta)}\left(\eta, \eta_{0}\right)} .
\end{aligned}
$$

By taking $\alpha$-integration from $\eta_{1}$ to $\eta$, we obtain

$$
\begin{aligned}
& \underset{\eta \rightarrow \infty}{\limsup } \int_{\eta_{1}}^{\eta}\left[\frac{E v(\kappa) z(\kappa)}{e_{R(\kappa)}\left(\sigma(\kappa), \eta_{0}\right)}-\frac{\ell(\kappa)\left(z^{\left(\Delta^{\alpha}\right)}(\kappa)\right)^{2}}{4 z(\kappa) e_{R(\eta)}\left(\kappa, \eta_{0}\right)}\right] \Delta^{\alpha} \kappa \\
& \quad \leq w\left(\eta_{1}\right)-w(\eta) \leq w\left(\eta_{1}\right) \leq \infty,
\end{aligned}
$$

which is a contradiction to (22). Similarly, we get a contradiction while assuming that $\chi(\xi, \eta)$ is the ENS of (1) and (2).

Remark 1. In Theorem 1, (18) can be replaced by

$$
\begin{aligned}
\limsup _{\eta \rightarrow \infty} & \frac{1}{\Lambda(\eta, H)} \int_{H}^{\eta} \Lambda(\eta, \kappa) \\
\quad & {\left[\frac{v(\kappa) z(\kappa)}{e_{R(\kappa)}\left(\sigma(\kappa), \eta_{0}\right)}-\frac{\ell(\kappa)\left(z^{\left(\Delta^{\alpha}\right)}(\kappa)\right)^{2}}{4 N z(\kappa) e_{R(\eta)}\left(\kappa, \eta_{0}\right)}\right] \Delta^{\alpha} \kappa=\infty, }
\end{aligned}
$$

for $\Lambda \in \Lambda$

By Theorem 1, we have

$$
w^{\left(\Delta^{\alpha}\right)}(\eta) \leq \frac{-v(\eta) z(\eta)}{e_{R(\eta)}\left(\sigma(\eta), \eta_{0}\right)}+\frac{\ell(\eta)\left(z^{\left(\Delta^{\alpha}\right)}(\eta)\right)^{2}}{4 N z(\eta) e_{R(\eta)}\left(\eta, \eta_{0}\right)} .
$$

Multiplying the above inequality by $\Lambda(\eta, \kappa)$ and taking $\alpha$-integration from $H$ to $\eta$, we get

$$
\begin{aligned}
& \int_{H}^{\eta} \Lambda(\eta, \kappa) w^{\left(\Delta^{\alpha}\right)}(\kappa) \Delta^{\alpha} \kappa \leq \int_{H}^{\eta} \Lambda(\eta, \kappa) \\
& \cdot\left[\frac{-v(\kappa) z(\kappa)}{e_{R(\kappa)}\left(\sigma(\kappa), \eta_{0}\right)}+\frac{\ell(\kappa)\left(z^{\left(\Delta^{\alpha}\right)}(\kappa)\right)^{2}}{4 N z(\kappa) e_{R(\eta)}\left(\kappa, \eta_{0}\right)}\right] \Delta^{\alpha} \kappa .
\end{aligned}
$$

Using integration by parts, we obtain

$$
\begin{aligned}
& {[\Lambda(\eta, \kappa) w(\eta)]_{H}^{\eta}-\int_{H}^{\eta} \Lambda^{\left(\Delta^{\alpha}\right)}(\eta, \kappa) w(\sigma(\kappa)) \Delta^{\alpha} \kappa} \\
& \quad \leq \int_{H}^{\eta} \Lambda(\eta, \kappa)\left[\frac{-v(\kappa) z(\kappa)}{e_{R(\kappa)}\left(\sigma(\kappa), \eta_{0}\right)}+\frac{\ell(\kappa)\left(z^{\left(\Delta^{\alpha}\right)}(\kappa)\right)^{2}}{4 N z(\kappa) e_{R(\eta)}\left(\kappa, \eta_{0}\right)}\right] \Delta^{\alpha} \kappa .
\end{aligned}
$$

By using the definition of the function $\Lambda$ and rearranging the terms in the above inequality, we have

$$
\frac{1}{\Lambda(\eta, H)} \int_{H}^{\eta} \Lambda(\eta, \kappa)\left[\frac{v(\kappa) z(\kappa)}{e_{R(\kappa)}\left(\sigma(\kappa), \eta_{0}\right)}-\frac{\ell(\kappa)\left(z^{\left(\Delta^{\alpha}\right)}(\kappa)\right)^{2}}{4 N z(\kappa) e_{R(\eta)}\left(\kappa, \eta_{0}\right)}\right] \Delta^{\alpha} \kappa \leq w(H) \leq \infty,
$$


which contradicts (26). Likewise, we obtain a contradiction when we take $\chi(\xi, \eta)$ is the ENS of (1) and (2).
Similarly, (22) can be replaced by the following in Theorem 2:

$$
\limsup _{\eta \rightarrow \infty} \frac{1}{\Lambda(\eta, H)} \int_{H}^{\eta} \Lambda(\eta, \kappa)\left[\frac{E v(\kappa) z(\kappa)}{e_{R(\kappa)}\left(\sigma(\kappa), \eta_{0}\right)}-\frac{\ell(\kappa)\left(z^{\left(\Delta^{\alpha}\right)}(\kappa)\right)^{2}}{4 z(\kappa) e_{R(\eta)}\left(\kappa, \eta_{0}\right)}\right] \Delta^{\alpha} \kappa=\infty,
$$

for $\Lambda \in \Lambda$.

\section{Oscillation of (1) with Boundary Condition (3)}

In this section, we use the eigenvalues of the Laplacian, $-\nabla^{2} \phi(\xi)=\lambda \phi(\xi)$ in $G, \phi(\xi)=0$ on $\partial G$. The principal eigenvalue $\lambda_{1}$ is positive, and the corresponding eigenfunction $\phi_{1}$ is also positive in the interior of G; see [26], Theorem 2, page 356 . Furthermore, we normalize this eigenvector so that $\int_{G} \phi_{1}=1$.

Lemma 5. If $\chi(\xi, \eta)$ is an EPS of (1) and (3), then there exists $\eta_{1} \geq \eta_{0}$ such that $\Upsilon_{1}(\eta)=\int_{G} \chi(\xi, \eta) \phi_{1}(\xi) d \xi>0$ and

$$
\begin{gathered}
{\left[\ell(\eta) \Upsilon_{1}^{\left(\Delta^{\alpha}\right)}(\eta)\right]^{\left(\Delta^{\alpha}\right)}+\hbar(\eta) \Upsilon_{1}^{\left(\Delta^{\alpha}\right)}(\eta)} \\
+v(\eta) f\left(\Upsilon_{1}(\eta)\right) \leq 0, \quad \forall \eta \geq \eta_{1} .
\end{gathered}
$$

Also, if $\chi(\xi, \eta)$ is an ENS, then there exists $\eta_{1} \geq \eta_{0}$ such that $\Upsilon_{1}(\eta)<0$ and

$$
\begin{aligned}
& {\left[\ell(\eta) \Upsilon_{1}^{\left(\Delta^{\alpha}\right)}(\eta)\right]^{\left(\Delta^{\alpha}\right)}+\hbar(\eta) \Upsilon_{1}^{\left(\Delta^{\alpha}\right)}(\eta)} \\
& \quad+v(\eta) f\left(\Upsilon_{1}(\eta)\right) \geq 0, \quad \forall \eta \geq \eta_{1} .
\end{aligned}
$$

Proof. Since $\chi(\xi, \eta)$ is an EPS of (1) satisfying (3), there exists $\eta_{1} \geq \eta_{0}$ such that $\chi(\xi, \eta) \geq 0 . \phi_{1}(\xi)>0$ in the interior of $\Omega$, which implies that $\Upsilon_{1}(\eta)=\int_{\Omega} \chi(\xi, \eta) \phi_{1}(\xi) \mathrm{d} \xi>0$. Multiply (1) by $\phi_{1}(\xi)$, and integrate in connection with $\xi$ over $\Omega$; then,

$$
\begin{aligned}
\int_{\Omega}\left(\ell(\eta) \chi^{\left(\Delta^{\alpha}\right)_{\eta}}(\xi, \eta)\right)^{\left(\Delta^{\alpha}\right)_{\eta}} \phi_{1}(\xi) \mathrm{d} \xi \\
\quad+\int_{\Omega} \hbar(\eta) \chi^{\left(\Delta^{\alpha}\right)_{\eta}}(\xi, \eta) \phi_{1}(\xi) \mathrm{d} \xi \\
\quad+\int_{\Omega} v(\xi, \eta) f(\chi(\xi, \eta)) \phi_{1}(\xi) \mathrm{d} \xi \\
=\int_{\Omega} \tau(\eta) \nabla_{\xi}^{2} \chi(\xi, \eta) \phi_{1}(\xi) d \xi .
\end{aligned}
$$

On the right-hand side of (34), we have

$$
\begin{aligned}
& \int_{\Omega} \tau(\eta) \nabla_{\xi}^{2} \chi(\xi, \eta) \phi_{1}(\xi) \mathrm{d} \xi \\
& \quad=-\lambda \tau(\eta) \int_{\Omega} \chi(\xi, \eta) \phi_{1}(\xi) \mathrm{d} \xi=-\lambda \tau(\eta) \Upsilon_{1}(\eta) \leq 0,
\end{aligned}
$$

which implies that

$$
\begin{aligned}
& \int_{\Omega}\left(\ell(\eta) \chi^{\left(\Delta^{\alpha}\right)_{\eta}}(\xi, \eta)\right)^{\left(\Delta^{\alpha}\right)_{\eta}} \phi_{1}(\xi) \mathrm{d} \xi \\
& \quad+\int_{\Omega} \hbar(\eta) \chi^{\left(\Delta^{\alpha}\right)_{\eta}}(\xi, \eta) \phi_{1}(\xi) \mathrm{d} \xi \\
& \quad+\int_{\Omega} v(\xi, \eta) f(\chi(\xi, \eta)) \phi_{1}(\xi) \mathrm{d} \xi \leq 0 .
\end{aligned}
$$

Applying Jensen's inequality and condition on $v$ to the third term of the above inequality and using Lemma 1 and continuity of $\left(\ell(\eta) \chi^{\left(\Delta^{\alpha}\right)_{\eta}}(\xi, \eta)\right)^{\left(\Delta^{\alpha}\right)_{\eta}}$ in the first term of the above inequality, we obtain

$$
\left[\ell(\eta) \Upsilon_{1}^{\left(\Delta^{\alpha}\right)}(\eta)\right]^{\left(\Delta^{\alpha}\right)}+\hbar(\eta) \Upsilon_{1}^{\left(\Delta^{\alpha}\right)}(\eta)+v(\eta) f\left(\Upsilon_{1}(\eta)\right) \leq 0
$$

A similar proof will be used for the ENS to obtain (33).

The following lemmas and theorems consider the solution $\Upsilon_{1}(\eta)$ of (1) and (3), and the proofs of the following are similar to those of lemmas and theorems proved for the solution $\Upsilon(\eta)$ of (1) and (2). Thus, we omit the proofs.

Lemma 6. Assume that $\int_{\eta}^{\infty} \Delta^{\alpha} \kappa / \ell(\kappa)=\infty$ and $R(\eta) \in \mathscr{R}_{+}$. If (32) has an EPS $\Upsilon(\eta)$, then there is sufficiently large $\eta_{1} \in\left[\eta_{0}, \infty\right)_{\Theta}$ such that $\left[\ell(\eta) Y_{1}^{\left(\Delta^{\alpha}\right)}(\eta) / e_{R(\eta)}\left(\eta, \eta_{0}\right)\right]^{\left(\Delta^{\alpha}\right)}<0$ and $Y_{1}^{\left(\Delta^{\alpha}\right)}(\eta)>0$ on $\left[\eta_{1}, \infty\right)_{\Theta}$.

Lemma 7. Assume that $\int_{\eta_{0}}^{\infty} \Delta^{\alpha} \kappa / \ell(\kappa)=\infty$ and $R(\eta) \in \mathscr{R}_{+}$. If (33) has an $\operatorname{ENS} \Upsilon_{1}(\eta)$, then there is sufficiently large $\eta_{1} \in\left[\eta_{0}, \infty\right)_{\Theta}$ such that $\left[\ell(\eta) Y_{1}^{\left(\Delta^{\alpha}\right)}(\eta) / e_{R(\eta)}\left(\eta, \eta_{0}\right)\right]^{\left(\Delta^{\alpha}\right)}>0$ and $Y_{1}^{\left(\Delta^{\alpha}\right)}(\eta)<0$ on $\left[\eta_{1}, \infty\right)_{\Theta}$.

Theorem 3. Assume that $\int_{\eta}^{\infty} \Delta^{\alpha} \kappa / \ell(\kappa)=\infty, R(\eta) \in \mathscr{R}_{+}$, and $f^{\prime}$ exists with $f^{\prime}(\eta) \geq N$ for some $N \geq 0, \forall \xi \neq 0$. If there exists $\psi(\eta)>0$ on $[H, \infty)_{\Theta}$ such that

$\limsup _{\eta \rightarrow \infty} \int_{H}^{\eta}\left[\frac{v(\kappa) \psi(\kappa)}{e_{R(\kappa)}\left(\sigma(\kappa), \eta_{0}\right)}-\frac{\ell(\kappa)\left(\psi^{\left(\Delta^{\alpha}\right)}(\kappa)\right)^{2}}{4 N \psi(\kappa) e_{R(\eta)}\left(\kappa, \eta_{0}\right)}\right] \Delta^{\alpha} \kappa=\infty$,

then (1) and (3) are oscillatory.

Theorem 4. Assume that $\int_{\eta}^{\infty} \Delta^{\alpha} \kappa / \ell(\kappa)=\infty, R(\eta) \in \mathscr{R}_{+}$, and $f(\xi) \geq$ Ex for some $E>0, \forall \xi \neq 0$. If there exists $\psi(\eta)>0$ on $[H, \infty)_{\Theta}$ such that 
$\limsup _{\eta \rightarrow \infty} \int_{H}^{\eta}\left[\frac{E v(\kappa) \psi(\kappa)}{e_{R(\kappa)}\left(\sigma(\kappa), \eta_{0}\right)}-\frac{\ell(\kappa)\left(\psi^{\left(\Delta^{\alpha}\right)}(\kappa)\right)^{2}}{4 \psi(\kappa) e_{R(\eta)}\left(\kappa, \eta_{0}\right)}\right] \Delta^{\alpha} \kappa=\infty$,

then (1) and (3) are oscillatory.

Remark 2. In Theorem 3, (38) can be replaced by

$$
\begin{aligned}
\limsup _{\eta \rightarrow \infty} & \frac{1}{\Lambda(\eta, H)} \int_{H}^{\eta} \Lambda(\eta, \kappa) \\
\cdot & {\left[\frac{v(\kappa) \psi(\kappa)}{e_{R(\kappa)}\left(\sigma(\kappa), \eta_{0}\right)}-\frac{\ell(\kappa)\left(\psi^{\left(\Delta^{\alpha}\right)}(\kappa)\right)^{2}}{4 N \psi(\kappa) e_{R(\eta)}\left(\kappa, \eta_{0}\right)}\right] \Delta^{\alpha} \kappa } \\
=\infty & \text { for } \Lambda \in \Lambda .
\end{aligned}
$$

Similarly, (39) can be replaced by the following in Theorem 4:

$$
\begin{aligned}
\limsup _{\eta \rightarrow \infty} & \frac{1}{\Lambda(\eta, H)} \int_{H}^{\eta} \Lambda(\eta, \kappa) \\
\cdot & {\left[\frac{E v(\kappa) \psi(\kappa)}{e_{R(\kappa)}\left(\sigma(\kappa), \eta_{0}\right)}-\frac{\ell(\kappa)\left(\psi^{\left(\Delta^{\alpha}\right)}(\kappa)\right)^{2}}{4 N \psi(\kappa) e_{R(\eta)}\left(\kappa, \eta_{0}\right)}\right] \Delta^{\alpha} \kappa } \\
=\infty & \text { for } \Lambda \in \Lambda .
\end{aligned}
$$

\section{Examples}

The equations considered below are an illustration to validate our established results.

Example 1. Consider

$$
\begin{gathered}
\left(\eta^{-1} \chi^{\left(\Delta^{0.5}\right)_{\eta}}(\xi, \eta)\right)^{\left(\Delta^{0.5}\right)_{\eta}}+\eta^{-3} \chi^{\left(\Delta^{0.5}\right)_{\eta}}(\xi, \eta) \\
+\eta^{-1} \chi(\xi, \eta)(1+|\chi(\xi, \eta)|)=\eta \nabla_{\xi}^{2} \chi(\xi, \eta),
\end{gathered}
$$

with $\chi_{x}(0, \eta)=\chi_{x}(\pi, \eta)=0$. Here, $\Theta=\mathbb{R} ; \Omega=(0, \pi) ; \alpha=$ $0.5 ; \ell(\eta)=\eta^{-1} ; \hbar(\eta)=\eta^{-3} ; f(\chi(\xi, \eta))=\chi(\xi, \eta)(1+\mid \chi(\xi$, $\eta) \mid) ; v(\eta)=\eta^{-1}$; and $\eta_{0}=1$. It is lucid that $f(\chi) \geq \chi \Rightarrow E=1$, $\sigma(\eta)=\eta, \mu(\eta)=0, R(\eta)=-\eta^{-(5 / 2)}$,

$$
\begin{array}{r}
\int_{\eta_{0}}^{\infty} \frac{\Delta^{\alpha} \kappa}{\ell(\kappa)}=\int_{\eta_{0}}^{\infty} \frac{\kappa^{-(1 / 2)} d \kappa}{\kappa^{-1}}=\int_{\eta_{0}}^{\infty} \kappa^{1 / 2} d \kappa=\infty, \\
1 \geq e_{R(\eta)}\left(\eta, \eta_{0}\right)=\exp \left(\int_{\eta_{0}}^{\eta} R(\kappa) d \kappa\right) \geq 1+\int_{1}^{\eta} R(\kappa) d \kappa \\
=1-\int_{1}^{\eta} \kappa^{-(5 / 2)} d \kappa=1+\frac{2}{3}\left(\eta^{-(3 / 2)}-1\right) \geq \frac{1}{3} .
\end{array}
$$

Let $z(\eta)=\eta^{1 / 2}$. Then, every condition of Theorem 2 is fulfilled. Hence, (42) with the given boundary condition is oscillatory.

\section{Example 2. Consider}

$$
\begin{aligned}
& \left(\eta^{-1 / 2} \chi^{\left(\Delta^{0.5}\right)_{\eta}(\xi, \eta)}\right)^{\left(\Delta^{0.5}\right)_{\eta}}+\eta^{-5 / 2} \chi^{\left(\Delta^{0.5}\right)_{\eta}}(\xi, \eta) \\
& +\eta^{-1} \chi(\xi, \eta)\left(E+e^{\chi(\xi, \eta)}\right)=\eta \nabla_{\xi}^{2} \chi(\xi, \eta),
\end{aligned}
$$

with $\chi(0, \eta)=\chi(\pi, \eta)=0$. Here, $\eta \in[2, \infty)_{\mathbb{Z}} ; \Theta=\mathbb{Z} ; \Omega=$ $(0, \pi) ; \alpha=0.5 ; \ell(\eta)=\eta^{-(1 / 2)} ; \hbar(\eta)=\eta^{-(5 / 2)} ; v(\eta)=\eta^{-1} ;$ $\eta_{0}=2$; and $f(\chi(\xi, \eta))=(\chi(\xi, \eta))^{\gamma}\left(E+e^{\chi(\xi, \eta)}\right)$.

Therefore, we get $f(\chi) \geq E \chi, \sigma(\eta)=\eta+1, \mu(\eta)=1$, $R(\eta)=-\eta^{-(5 / 2)}$, and $1+\mu(\eta) R(\eta)=1-\eta^{-(5 / 2)} \geq 1-(1 /$ 2) $>0$ which implies $R(\eta) \in \mathscr{R}^{+}$. Also,

$$
\begin{aligned}
\int_{\eta_{0}}^{\infty} \frac{\Delta^{\alpha} \kappa}{\ell(\kappa)} & =\int_{2}^{\infty} \frac{\kappa^{\alpha-1} \Delta \kappa}{\ell(\kappa)}=\sum_{\kappa=2}^{\infty} \frac{\kappa^{\alpha-1}}{\kappa^{-(1 / 2)}}=\sum_{\kappa=2}^{\infty} 1=\infty \\
1>e_{R(\eta)}\left(\eta, \eta_{0}\right) & =\exp \left(\int_{\eta_{0}}^{\eta} R(\kappa) \Delta \kappa\right) \geq 1+\int_{2}^{\eta} R(\kappa) \Delta \kappa=1-\sum_{\kappa=2}^{\eta-1} \kappa^{-(5 / 2)} \\
& \geq 1-\int_{1}^{\eta-1} \kappa^{-(5 / 2)} \mathrm{d} \kappa=1+\frac{2}{3}\left((\eta-1)^{-(3 / 2)}-1\right)>\frac{1}{3}
\end{aligned}
$$

Let $\psi(\eta)=\eta$. Then, all the conditions of Theorem 4 are fulfilled. Hence, (44) with the given boundary condition is oscillatory.

\section{Conclusion}

In this article, we established the oscillation criteria for the fractional partial dynamic equation on time scales. The obtained results are improved in the sense that which provide sufficient criteria for the oscillation of the considered equation with two boundary conditions. Finally, numerical examples are also presented to validate the theoretical results of this study. Additionally, more complex systems, including the fractional dynamic equation with time delay, are interesting research topics and will be examined in our future works.

\section{Data Availability}

No data were used to support this study. 


\section{Conflicts of Interest}

The authors declare that there are no conflicts of interest regarding the publication of this paper.

\section{Acknowledgments}

The third author was supported by the Department of Science and Technology, New Delhi, India, under the FIST Programme (SR/FST/MS1-115/2016). This research was supported by the National Research Foundation of Korea (NRF) grant funded by the Korea government (MSIT) (no. 2021R1F1A1048937) to the last author.

\section{References}

[1] R. Khalil, M. Al Horani, A. Sababheh, and M. Sababhed, "A new definition of fractional derivative," Journal of Computational and Applied Mathematics, vol. 264, pp. 65-70, 2014.

[2] D. R. Anderson and D. J. Ulness, "Properties of the Katugampola fractional derivative with potential application in quantum mechanics," Journal of Mathematical Physics, vol. 56, no. 6, pp. 1-18, 2015.

[3] H. Batarfi, J. Losada, J. J. Nieto, and W. Shammakh, "ThreePoint boundary value problems for conformable fractional differential equations," Journal of Function Spaces, vol. 20156 pages, Article ID 706383, 2015.

[4] R. P. Agarwal, M. Bohner, T. Li, and C. Zhang, "Oscillation criteria for second-order dynamic equations on time scales," Applied Mathematics Letters, vol. 31, pp. 34-40, 2014.

[5] C. D. Ahlbrandt and C. Morian, "Partial differential equations on time scales," Journal of Computational and Applied Mathematics, vol. 141, no. 1-2, pp. 35-55, 2002.

[6] D. R. Anderson, "Oscillation of second-order forced functional dynamic equations with oscillatory potentials," Journal of Difference Equations and Applications, vol. 13, no. 5, pp. 407-421, 2007.

[7] M. Bohner, L. Erbe, and A. Peterson, "Oscillation for nonlinear second order dynamic equations on a time scale," Journal of Mathematical Analysis and Applications, vol. 301, no. 2, pp. 491-507, 2005.

[8] M. Bohner and S. H. Saker, "Oscillation of second order nonlinear dynamic equations on time scales," Rocky Mountain Journal of Mathematics, vol. 34, pp. 1239-1254, 2004.

[9] L. Erbe, A. Peterson, and S. H. Saker, "Oscillation criteria for second order nonlinear dynamic equations on time scales," Journal of the London Mathematical Society, vol. 67, no. 3, pp. 701-714, 2003.

[10] L. Erbe, A. Peterson, and S. H. Saker, "Oscillation criteria for second-order nonlinear delay dynamic equations," Journal of Mathematical Analysis and Applications, vol. 333, no. 1, pp. 505-522, 2007.

[11] Q. Feng and F. Meng, "Oscillation results for a fractional order dynamic equation on time scales with conformable fractional derivative," Advances in Difference Equations, vol. 2018, no. 1, p. 193, 2018.

[12] Q. Qinghua Feng, "Oscillatory and asymptotic criteria of third order nonlinear delay dynamic equations with damping term on time scales," Journal of Applied Analysis \& Computation, vol. 8, no. 4, pp. 1260-1281, 2018.

[13] T. Li and S. H. Saker, "A note on oscillation criteria for second-order neutral dynamic equations on isolated time scales," Communications in Nonlinear Science and Numerical Simulation, vol. 19, no. 12, pp. 4185-4188, 2014.

[14] W. Weinian Li, W. Sheng, P. Weihong Sheng, and P. Zhang, "Oscillatory properties of certain nonlinear fractional nabla difference equations," Journal of Applied Analysis \& Computation, vol. 8, no. 6, pp. 1910-1918, 2018.

[15] P. Prakash, S. Harikrishnan, and M. Benchohra, "Oscillation of certain nonlinear fractional partial differential equation with damping term," Applied Mathematics Letters, vol. 43, pp. 72-79, 2015.

[16] S. H. Saker, "Oscillation criteria of second-order half-linear dynamic equations on time scales," Journal of Computational and Applied Mathematics, vol. 177, no. 2, pp. 375-387, 2005.

[17] S. H. Saker, R. P. Agarwal, and D. O'Regan, "Oscillation of second order damped dynamic equations on time scales," Journal of Mathematical Analysis and Applications, vol. 330, pp. 1317-1337, 2004.

[18] Y. Ying Sui and Z. Zhenlai Han, "Oscillation of second order nonlinear dynamic equations with a nonlinear neutral term on time scales," Journal of Applied Analysis \& Computation, vol. 8, no. 6, pp. 1811-1820, 2018.

[19] S. Sun, Z. Han, and C. Zhang, "Oscillation of second order delay dynamic equations on time scales," Journal of Applied Mathematics and Computing, vol. 30, no. 1-2, pp. 459-468, 2009.

[20] J. Jiangfeng Wang and F. Fanwei Meng, "Oscillatory behavior of a fractional partial differential equation," Journal of Applied Analysis \& Computation, vol. 8, no. 3, pp. 1011-1020, 2018.

[21] M. Bohner and G. S. Guseinov, "Partial differentiation on time scales," Dynamic Systems and Applications, vol. 13, pp. 351379, 2004.

[22] J. Hoffacker, "Basic partial dynamic equations on time scales," Journal of Difference Equations and Applications, vol. 8, no. 4, p. 307, 2002.

[23] B. Jackson, "Partial dynamic equations on time scales," Journal of Computational and Applied Mathematics, vol. 186, no. 2, pp. 391-415, 2006.

[24] N. Benkhettou, S. Hassani, and D. F. M. Torres, "A conformable fractional calculus on arbitrary time scales," Journal of King Saud University Science, vol. 28, no. 1, pp. 93-98, 2016.

[25] M. Bohner and A. Peterson, Dynamic Equations on Time Scale, an Introduction with Applications, Birkhäuser, Basel, Switzerland, 2001.

[26] L. C. Evans, "Partail differential equations," American Math Society, Graduate Studies in Mathematics, vol. 19, 2010. 